Proc. Indian Acad. Sci. (Chem. Sci.), Vol. 96, No. 6, April 1986, pp. 465-472. (C) Printed in India.

\title{
On the kinetics of crystal growth from a supercooled melt $\dagger$
}

\author{
BIMAN BAGCHI* and T R KIRKPATRICK $\$$ \\ Solid State and Structural Chemistry Unit, Indian Institute of Science, Bangalore 560012. \\ India \\ $\S$ Institute for Physical Science and Technology and Department of Physics and Astronomy, \\ University of Maryland, College Park, MD 20742, USA
}

\begin{abstract}
We present a theoretical analysis of the dynamics of crystal growth from a supercooled meit. A molecular theory of crystal growth that pays proper attention to the structure at the liquid-solid interface is discussed.
\end{abstract}

Keywords. Liquid-solid transition; crystal growth; supercooled melt; order parameters; diffusion equation; structure factor; heat mode.

\section{Introduction}

Kinetics of crystal growth has been a subject of absorbing interest for several decades (Cahn 1960; Cahn et al 1964; Bennema and Gilmer 1973; Carruthers 1979; Langer 1980). Recently attention has been focussed on understanding the dynamics of crystal growth from a microscopic basis. In this article we present a molecular theory of crystal growth developed by Bagchi and Kirkpatrick (1986) and discuss some of its consequences. In the next section we discuss the structure at the liquid-solid interface and its relevance to the kinetics of crystal growth. In $\$ 3$ we describe the recent theory of Bagchi and Kirkpatrick. Section 4 concludes with a discussion.

\section{Structure at the liquid-solid interface}

Despite considerable effort over the years, the structure of the liquid-solid interface remains controversial and poorly understood. The main reason for this uncertainty is that it is difficult to obtain experimental information about the interface which is quite narrow and is bounded by two condensed phases. Most of our understanding on the structure of the interface comes from computer simulations (Cape and Woodcock 1980; Broughton et al 1981; Cleveland et al 1982) and from theoretical studies (Cahn and Kikuchi 1985; Haymet and Oxtoby 1981; Oxtoby and Haymet 1983; Tempkin 1966). Computer simulation studies indicate that the liquid-solid interface of a simple one-component system may be 6-8 monolayers wide. The decay of crystalline order may depend on the orientation of the crystalline solid. Theoretical investigations have reached similar conclusions on the structure of the interface. In the following we briefly discuss two theoretical studies that are relevant to the present work.

† Contribution number 346 from the Unit.

* To whom all correspondence should be addressed. 
The first study we discuss is that of Tempkin (1966) who proposed a multi-layer model of the interface. The merit of the Tempkin model is that it does not limit the number of layers and that it can be applied to different kinds of interfaces. This model consists of an infinite number of layers with the thickness $d_{h k l}$, the interplanar distance of the $(h k l)$ face. Molecules in each layer are divided into solid-like and liquid-like molecules. A solid-like molecule can occur only on another solid molecule. The fraction of solid molecules in the $n$th layer, $C_{n}$, is the order parameter in this model. In the Tempkin model, $n$ ranges from $-\infty$ (solid) to $+\infty$ (liquid) with $C_{-\infty}=1$ and $C_{+\infty}=0$. The assumption of solid on solid (sos) rules out 'overhangs' and implies that $C_{n+1} \leqslant C_{n}$. In the Tempkin formalism one calculates the change in Gibbs free energy that occurs if a completely fiat 'that is, singular) interface is 'roughened'. For the flat reference face the following conditions hold: $-\infty<n<0, C_{n}=1$, and $1<n<+\infty, C_{n}=0$; the reference face is placed between $n=0$ and $n=1$.

By using standard methods of statistical thermodynamics, Tempkin obtained the following expression for $\Delta G$, the change of Gibbs free energy upon roughening a singular reference plane for the (001) face of a simple cubic lattice:

where

$$
\begin{aligned}
\frac{\Delta G}{N k_{B} T}= & \beta\left[\sum_{n=-\infty}^{0}\left(1-C_{n}\right)-\sum_{n=1}^{\infty} C_{n}\right] \\
& +\alpha \sum_{n=-\infty}^{\infty} C_{n}\left(1-C_{n}\right)+\sum_{n=-\infty}^{\infty}\left(C_{n}-C_{n+1}\right) \ln \left(C_{n}-C_{n+1}\right)
\end{aligned}
$$

$$
\alpha=4 \varepsilon / k_{B} T, \quad \beta=\Delta \mu / k_{B} T .
$$

$2 \varepsilon$ is the energy gain upon formation of two solid-liquid bonds by replacing a solid-solid bond and a liquid-liquid bond. $\Delta \mu$ is the change in chemical potential where a solid-like molecule is converted into a liquid-like molecule, i.e. $\Delta \mu$ is the difference in chemical potential if the two phases are not in thermodynamic equilibrium. $k_{B}$ is the Boltzman constant and $T$ the temperature. A self-consistent equation can be obtained from (1) by minimizing $\Delta G$ with respect to $C_{n}$. This leads to the following equation:

$$
\left[\left(C_{n}-C_{n+1}\right) /\left(C_{n-1}-C_{n}\right)\right] \exp \left(-2 \alpha C_{n}\right)=\exp (-\alpha+\beta),
$$

which has to be solved numerically. Main results of Tempkin model are summarized below:

(i) The interface is sharp for high values of $\alpha$ and is rough and broad for low $\alpha$. There is no 'roughening' transition as $\alpha$ is lowered, but the interface can be considered rough for $\alpha \lesssim 1$ and sharp (or flat or singular) for $\alpha \gtrsim 4$. For most metals $\alpha \simeq 1$ and so the interface is rough.

(ii) For nonequilibrium interfaces with $\beta \neq 0$, the Tempkin model predicts two regions in the $\beta-\alpha$ plane separated by a continuous line originating at $\beta=0, \alpha \simeq 1 \cdot 1$. In the region with higher $\alpha$ and lower $\beta$, there is a stable interface, whereas in the other region there is no stable solution to (3). In the latter region, Tempkin's model predicts continuous growth for infinitesimal $\beta$. For example, for most metals, Tempkin's model predicts continuous growth even for $\beta \simeq 10^{-5}$. For organic crystals like salol with high $\alpha$, a large driving force is necessary to maintain growth of the crystal. It is worth noting that $\alpha$ is proportional to the latent heat of fusion.

Despite the considerable success of Tempkin's model, it suffers from some fundamental drawbacks when applied to the crystal-melt interface. For a crystal-melt 
interface it is not possible to discriminate between a solid-like and a liquid-like molecule. Another drawback is its failure to describe the semi-crystalline topological order present at the interface; $C_{n}$ is just an average density. A more detailed description is desirable.

Such a description has been provided recently (Haymet and Oxtoby 1981; Oxtoby and Haymet 1983) in a molecular theory of the crystal-melt interface by generalizing the order parameter description of freezing developed by Ramakrishnan and Yussouff (1979). The central point of the Haymet-Oxtoby formalism is that the interface is treated as a perturbation about the homogeneous liquid phase. The main assumption of this work is that the interface is sufficiently broad, compared to the molecular diameter, so that a square-gradient approximation on the spatial variation of the order parameters can be made. The order parameters describe the inhomogeneous density distribution at the interface which is assumed to be of the following form

$$
n_{0}(\mathbf{r})=\rho_{l}\left[1+\phi_{0}(\mathbf{r})\right]+\rho_{l} \sum_{n} \phi_{n}(\mathbf{r}) \exp (i \mathbf{G} \cdot \mathbf{r})
$$

where $\rho_{l}$ is the average equilibrium density of the liquid phase and the $\mathbf{G}$ are the reciprocal lattice vectors (RLV) of the crystalline solid phase. $\phi_{0}$ and $\phi_{n}$ are the order parameters that characterize the structure at the liquid-solid interface. They are analogues of the $C_{n}$ of Tempkin's model. These order parameters are zero in the pure liquid phase far from the interface and have constant non-zero values in the pure solid phase. It is assumed that these order parameters change smoothly from their liquid-like to solid-like values as the interface is traversed from liquid to solid.

Oxtoby and Haymet (1983) obtained a set of self-consistent differential equations for the variation of $\phi_{0}$ and $\phi_{n}$ and solved these equations numerically for planar bcc 100 and 111 crystal-melt interfaces. Their calculations show that the interfacial transition zone is quite broad, which is consistent with their starting assumption. One interesting prediction of the theoretical calculations is the existence of a 'structured liquid' region in the interface characterized by a liquid-like average density, but solid-like topological ordering.

It is obvious from the preceding discussion that the diffuse interface model of Haymet and Oxtoby (proposed originally by Cahn 1960) predicts a picture of the solidliquid interface that is considerably different from that of Tempkin. In Tempkin's model, the microscopic boundary between solid and liquid is sharp, even after the 'roughening' of the surface. Recent investigations of Weeks and coworkers (Weeks and Gilmer 1979) have clearly shown the presence of a roughening transition for a solidvapour interface. For a Lennard-Jones system, the roughening temperature, $T_{R}$, is below the triple point. So, this roughening transition may be of little relevance to a solid-liquid interface since the density of the liquid even at a temperature slightly above the triple point is at least an order of magnitude larger than that of the vapour. Thus, a description of the solid-liquid interface cannot be based on models which are primarily designed to describe a solid-vapour interface. A dense liquid is characterized by considerable amount of short range order. Any theory of the solid-liquid interface must pay attention to this local structure in dense liquids. This view may be further strengthened by looking into the disparate time scales that are involved in the dynamical processes at these two interfaces. At a solid-vapour interface, the selfdiffusion coefficient of a molecule in the solid phase is negligibly small compared to that 
in the vapour phase. Thus, for many properties, one can distinguish between a molecule in the solid and in the vapour state. Such a clear demarcation does not exist at a microscopic level for the solid-liquid interface; there is not sufficient separation in time scales of motion of a molecule in two adjacent layers. Thus, a diffuse interface model, in which a gradual change in atomic arrangements occurs over several molecular layers, seems more appropriate. This view finds support in results of recent computer simulations of solid-liquid interfaces.

In this paper we assumed a diffuse interface model to describe the dynamics of crystal growth from a supercooled melt. Our theory pays proper attention to the local structure of the liquid. A considerable amount of work need to be done before a detailed comparison of this theory with experiment can be made. We now briefly discuss the theory.

\section{A molecular theory}

We shall consider the growth of a perfect crystal from its supercooled melt. Because of supercooling, there will be a thermodynamic driving force towards solidification at the interface which will favour advancement of the crystalline front. In addition to supercooling, the velocity of crystal growth will be determined by two other factors: the rate of removal of the latent heat generated by solidification and the viscous forces present in the liquid which inhibit the formation of crystalline order. Thus the process of crystal growth is complicated due to the involvement of several dynamical processes of varying temporal and spatial dependences.

For a crystal to grow into the adjacent melt, several microscopic processes must occur simultaneously. Firstly, a transport of mass is involved because the crystal is denser than the liquid. For many solid-liquid transformations, the fractional density change involved is substantial, often $10-20 \%$ of the density of the liquid. Even for a much smaller fractional density change (as is the case for liquid metals), it may play an important role in crystal growth because it is a locally conserved variable and in a high density liquid, especially at the solid-liquid interface, transport of particles is a slow process. Secondly, the periodic arrangement of particles must build up from the spatially random liquid structure. An important point here is that the interface is rather narrow. Thus, the local structure of the liquid is important in the crystallization process. One can approximately distinguish between two regions within the interface. In the region close to the solid surface, the density and atomic arrangements will be solid-like, whereas in the region close to the liquid side, the opposite is true. For a slow steady growth of the crystal, these quantities (the density and the topological order) are expected to evolve slowly towards their solid phase values. In this limit, one can generalise the order parameters $\phi_{0}$ and $\phi_{n}$ of (4) to the time domain and expand the time dependent singlet distribution function in the following form:

$$
n(\mathbf{r}, t)=\rho_{l}\left[1+\phi_{0}(\mathbf{r}, t)\right]+\rho_{I} \sum_{n} \phi_{n}(\mathbf{r}, t) \exp (i \mathbf{G} \cdot \mathbf{r}) .
$$

As before, $\phi_{0}$ represents the time dependence of the fractional change in density, averaged over an unit cell of the lattice, and $\phi_{n}$ represents the time dependence of the oscillatory part in density. Such a separation is meaningful because the magnitudes of G's are large. 
It is worth pointing out that $\phi_{0}$ and $\phi_{n}$ are coupled. So, crystal growth is a process where a locally conserved variable $\left(\phi_{0}\right)$ (in a coarse-grained description) is coupled to a nonconserved variable $\left(\phi_{n}\right)$. In the terminology of time dependent statistical mechanics, such a process corresponds to the model $C$ of the time dependent Ginzburg-Landau (TDGL) theories discussed by Hohenberg and Halperin (1977). One objective of this work is to obtain equations of motions for $\phi_{0}$ and $\phi_{n}$ from a model kinetic equation for $n(r, t)$.

The kinetic equation proposed by Bagchi and Kirkpatrick (1986) is a non-linear diffusion equation that contains a mean-field force term due to the liquid structure present on small length scales and a term due to the temperature variation at the interface. The equation is given by

$$
\frac{\partial n(\mathbf{r}, t)}{\partial t}=\nabla \cdot \overrightarrow{\mathbf{D}}(\mathbf{r}, t) \cdot[\nabla n(\mathbf{r}, t)-\beta n \mathbf{F}]-\nabla \cdot \overrightarrow{\mathbf{D}} \cdot n \nabla \ln \beta,
$$

where $\overrightarrow{\mathbf{D}}(r, t)$ is the position and time dependent self-diffusion tensor, $\beta$ is $\left[k_{B} T(r, t)\right]^{-1}$ and $F(r, t)$ is the mean-field force term given by

$$
\beta F(r, t)=\nabla \int \mathrm{d} r^{\prime} \mathrm{c}\left(r-r^{\prime}\right) n\left(r^{\prime}, t\right),
$$

$c(r)$ is the two particle direct correlation function.

The justification for using a non-linear diffusion equation to describe the dynamics of crystal growth has been discussed elsewhere (Bagchi and Kirkpatrick 1986). Here we briefly mention the salient points. Firstly, (6) has correct limiting properties. At equilibrium, the solution of (6) is the standard mean-field expression for the inhomogeneous density distribution that has been used successfully in the theory of freezing. In a homogeneous system in the absence of a temperature gradient, (6) leads to an expression for the dynamic structure factor $S(k, \omega)$, which can describe neutron scattering by dense classical liquids fairly well for large values of the wave vector $\mathbf{k}$. Secondly, recent work of de Schepper and Cohen (1982), and also of Kirkpatrick (1985), on short wavelength collective modes show that the dynamics at large wave numbers are dominated by a self-diffusion-like mode alone. Thirdly, the mean free path in a liquid at freezing density is extremely small, only 3-5\% of molecular diameter. This last fact implies that in a dense liquid a hydrodynamic-like description can be used to describe processes taking place on a molecular scale.

Next, we substitute (5) for $n(r, t)$ in (7) to obtain an expression for $F(r, t)$. Equations $(5)$ and (6) are then combined to obtain an equation of motion for the order parameters. The resulting expression is rather complicated. We write it in the following form

$$
\begin{aligned}
\frac{\partial \phi_{0}}{\partial t}+ & \frac{\partial}{\partial t} \sum_{n} \phi_{n}(\mathbf{r}, t) \exp \left(i \mathbf{G}_{n} \cdot \mathbf{r}\right)=\left[A(\mathbf{r}, t)-\rho_{l} \sum_{n} Q_{n,-n}(\mathbf{r}, t)\right] \\
& +\rho_{l} \sum_{n} B_{n}(\mathbf{r}, t) \exp \left(i \mathbf{G}_{n} \cdot \mathbf{r}\right)-\rho_{l} \sum_{\substack{n, m \\
G_{n} \neq-G_{m}}} Q_{n, m}(\mathbf{r}, t) \exp \left[i\left(\mathbf{G}_{n}+\mathbf{G}_{m}\right) \cdot \mathbf{r}\right],
\end{aligned}
$$

where the coefficients $A, B_{n}$ and $Q_{n, m}$ are functions of the order parameters $\phi_{0}$ and $\phi_{G}$, of $D$ and $T$, and of their spatial derivatives. Expressions for $A, B_{n}$ and $Q_{n, m}$ are given elsewhere (Bagchi and Kirkpatrick 1986).

From (8), we can derive equations of motion for the order parameters $\phi_{0}$ and $\phi_{n}$ if we assume that $\phi_{0}$ and $\phi_{n}$ vary smoothly in space and time. The form of the resulting 
equations is similar to the ones obtained from the time dependent Ginzburg-Landau description, with one important difference. In our formalism, we have explicit expressions for the coefficients that occur in the expansion of the free energy functional in the order parameters. Another aspect of (8) is that it is derived from a kinetic equation (6) and so is expected to the valid in nonequilibrium situations.

Equation (8) must be supplemented with an equation of motion of the temperature field $T(r, t)$. In principle, one should be able to express the rate of change of $T(r, t)$ in terms of the rate of change of the order parameters, and the thermal diffusion coefficient. Collins and Levine (1985) have recently initiated such an approach to the problem of diffusion limited crystal growth. The basic idea here is that as the crystalline front advances into the liquid, the order parameters change from liquid-like to solidlike values giving rise to latent heat of fusion which must be removed from the interface by thermal diffusion. So, we should find an expression for the local latent heat of fusion (that is, a position dependent entropy change) in terms of the order parameters. We shall leave this taks for the future. In this paper we shall assume a linear temperature gradient across the interface and find an expression for the velocity of steady growth of a planar crystal surface.

Let us assume that the crystal is growing in the $z$-direction with a constant velocity $V$. In the coordinate frame attached to the moving surface, $\phi_{0}, \phi_{n}, D$ and $T$ will be approximately independent of time for a slowly advancing interface and their dependence on the position coordinate $z$ will be similar to that of a stationary equilibrium interface. The first term on the right hand side of (6) will then be small and negligible. Making the coordinate transformation

we obtain

$$
z_{1}=z+V t
$$

$$
\frac{\partial}{\partial t} n\left(z_{1}-V t\right)=\frac{\partial}{\partial z_{1}} D\left(z_{1}-V t\right) n\left(z_{1}-V t\right) \times \frac{1}{T} \frac{\partial}{\partial z_{1}} T\left(z_{1}-V t\right) .
$$

The time derivatives of the order parameters are given by

$$
\begin{gathered}
\frac{\partial}{\partial t} \phi_{0}\left(z_{1}-V t\right)=-V \frac{\partial \phi_{0}}{\partial z_{1}}, \\
\frac{\partial}{\partial t} \phi_{n}\left(z_{1}-V t\right)=-V \frac{\partial \phi_{n}}{\partial z_{1}} .
\end{gathered}
$$

Using (11) and (5) in (10) and keeping only the terms that are multiplied by $\left(\mathbf{G}_{n} \cdot 2\right)$ because they are the dominating terms, we obtain the following simple expression for $V$

$$
V=-\frac{1}{z_{w}} \int_{z_{s}}^{z_{1}} \mathrm{~d} z D(z) \frac{1}{T} \frac{\partial}{\partial z} T(z),
$$

where $z_{w}$ is the width of the interface, and $z_{1}$ and $z_{s}$ denote positions where the order parameters reach their liquid-like and solid-like values, respectively. If we further assume a linear dependence of $T(z)$ on $z$, we recover the usual linear dependence (Cahn 1960) of $V$ on $\Delta T$-the temperature difference between the crystal and the melt. 


\section{Discussion}

An analysis (Langer 1980) of the thermal diffusion equation with the usual equilibrium Joule-Thomson boundary condition shows that a sleady-state solution for the thermal field is possible only for a unique supercooling $\left(T(-\infty)=T_{m}-L / C_{p}\right.$ where $T_{m}$ is the equilibrium melting temperature, $L$ the latent heat and $C_{p}$ the specific heat). At this supercooling, the crystal growth velocity is arbitrary (Langer 1980). As discussed by Collins and Leyine (1985), this is simply because of the absence of any length scale in the problem. In the analysis of Bagchi and Kirkpatrick (1986), the length scale is determined by the variation of the order parameters in the interface and this gives rise to a well-defined velocity of growth of a planar interface, as given by (12).

It is interesting to compare our theory with Cahn's original (Cahn 1960) theory of crystal growth. The fundamental ideas are quite similar. The main difference is that our work is more microscopic and pays proper attention to the structure at the solid-liquid interface. This has been possible because of our use of the nonlinear diffusion equation (6) to describe the dynamics at the interface.

A limitation of this work is that it is based on a mean-field description of the potential experienced by the particles at the interface. However, the justification of using a meanfield description in the present problem comes from the following observations. Firstly, such a description has been successfully used in the theories of freezing. Secondly, it gives fairly accurate description of the dynamic structure factor, $S(k, w)$, for large values of $k$. Since dynamics of freezing involves variations on molecular length scales, a description at the level of the mean-field may be fairly accurate.

\section{Acknowledgement}

One of us (TRK) is the recipient of a presidential young investigator award (1984-1989).

\section{References}

Bagchi B and Kirkpatrick T R 1986 J. Chem. Phys. Dynamics of crystal growth from supercooled melt (submitted)

Bennema P and Gilmer G H 1973 in Crystal growth: An introduction (eds) W Bardsky, D T J Hurk and $J$ B Mullin (Amsterdam: North-Holland)

Broughton J Q, Bonissent A and Abraham F F $1981 \mathrm{~J}$. Chem. Phys. 744029

Cahn J W 1960 Acta Metall. 8554

Cahn J W, Hillig W B and Sears G W 1964 Acta Metall. 121421

Cahn J W and Kikuchi R 1985 Phys. Rev. B31 4300

Cape J N and Woadcock L V 1980 J. Chem. Phys. 732420

Carruthers J R 1979 in Crystal growth; A tutorial approach (eds) W Bardsley, D T J Hurle and J B Mullin (Amsterdam: North-Holland)

Cleveland C L, Landman U and Barnett R N 1982 Phys. Rev. Lett. 49790

Collins J B and Levine H 1985 Phys, Rev, B31 6119

de Schepper I M and Cohen E G D 1982 J. Stat. Phys. 27223

Hohenberg P and Halperin B I 1977 Rev. Mod. Phys. 49435

Haymet A D J and Oxtoby D W 1981 J. Chem. Phys. 742559

Kirkpatrick T R 1985 Phys. Rev. A32 3130

Langer J S 1980 Rev. Mod. Phys. 521 
Oxtoby D W and Haymet A D J 1983 J. Chem. Phys. 766262 Ramakrishnan T V and Yussouff M 1979 Phys. Rev. B19 2775

Tempkin D E 1966 in Crystallization processes (New York: Consultants Bureau) p. 15

Weeks J D and Gilmer G H 1979 Advances in Chemical Physics (eds) I Prigogine and S A Rice (New York: Wiley) 